\title{
Early detection and Multistage classification of Diabetic Retinopathy using Random Forest Classifier
}

\author{
Deepthi K Prasad \\ Full time research scholar, Department of CSE \\ B N M Institute of Technology, Bangalore, Karnataka, India \\ E-mail: deepthikp.bnmit@gmail.com \\ Vibha L \\ Professor, Department of CSE \\ B N M Institute of Technology, Bangalore, Karnataka, India \\ E-mail: vibhal1@rediffmail.com \\ Venugopal K R \\ Principal, UVCE, Bangalore University, Bangalore, India \\ E-mail: venugopalkr@gmail.com
}

\begin{abstract}
Diabetic Retinopathy (DR) is an injury to the retina due to uncontrolled blood sugar levels. Early detection and diagnosis of this disorder is essential as it may lead to complete vision loss if left untreated. In this work, a system for detecting the various stages of DR is proposed. Image morphological operations and Thresholding techniques are used for segmenting the retinal features like microaneurysms, hemorrhages. Large set of features are extracted from the images. Classification is performed using Random forest classifiers into four distinct stages namely normal, Mild -NPDR, Moderate- NPDR and PDR for publicly available data set. The accuracy obtained is $\mathbf{9 1 . 2 \%}$.
\end{abstract}

Keywords - Diabetic Retinopathy; Fundus Images; Microaneurysms; Hemorrhages; Exudates; Random Forest

\section{INTRODUCTION}

Diabetic retinopathy (DR) is an exacerbation of diabetes resulting in damage to the retinal blood vessels. It does not exhibit any symptoms at the initial stages but eventually results in blindness if not detected and diagnosed at earlier stage. Around 285million people in the world have vision problems world-wide according to the survey of World Health Organization (WHO) [11]. Figure 1 shows a sample input image affected with DR.

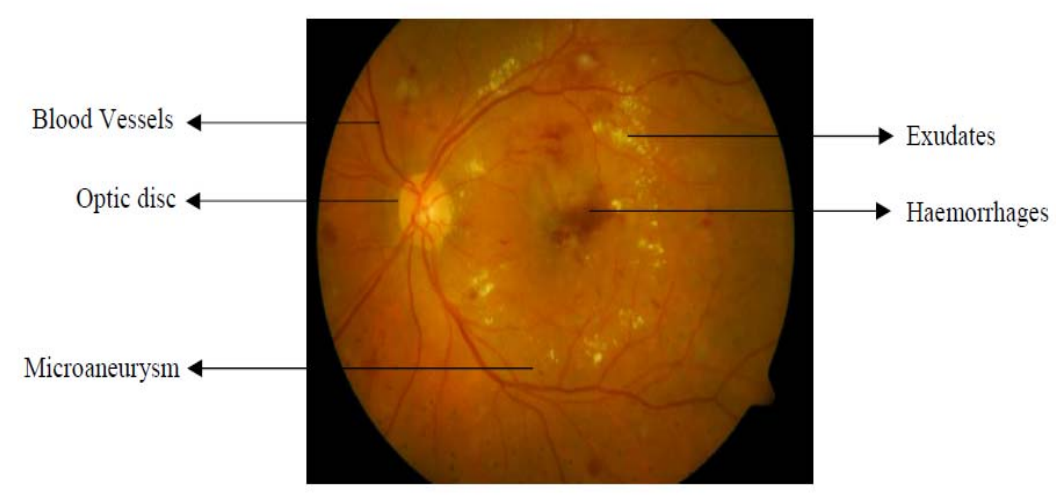

Figure 1 DR Affected Sample Retinal Image

DR initially is asymptotic. But as the disease progresses, various retinal abnormalities begin to appear inside the retina. Microaneurysms (MA) are the first visible signs of DR. They are formed when the blood vessels inside the retina become weak. MA appear as tiny red spots appear as bulges in the blood vessels and eventually leak plasma and other fluid into the retina. This fluid may also get deposited in the macula region of the retina and cause the macula to swell. These MA may rupture in to the deeper retinal layers and referred to as Hemorrhages. The blood vessels may also leak lipid and other protein deposits called Hard Exudates (HE). This stage is termed as Non Proliferative DR (NPDR). 
NPDR, if left untreated may progress to advanced stage known as Proliferative DR (PDR). In this stage, the retina doesn't get enough oxygen and fragile vessels emerge inside the retina and this is known as neovascularization. If PDR is also not treated, it would lead to retinal detachment and permanent vision loss.

Motivation: DR is one of the major reasons for blindness due to uncontrolled diabetes. It can be corrected if detected and treated at early stages. It is not possible for people in rural areas to regularly get the eyes checked for the progression as it is not economically feasible. So there is need for a mass screening system that can grade the retinal images based on the severity levels. Only those cases that need immediate attention may be referred to an ophthalmologist thus reducing the burden of analyzing bulk of images coming from these mass screening programmes. This would also be helpful in rural areas where there is shortage of experts and high end imaging equipments.

Contribution: The proposed system can be used extensively in rural areas for mass screening. It would classify the input images into one of the four categories namely, normal, mild-NPDR, moderate-NPDR and PDR using Random forest classifier with an accuracy of $90 \%$.

The flow of this paper is given below. Earlier and latest work in DR detection and classification is described in Section II. The proposed system is presented and described in Section III. Section IV describes the results of segmentation and severity classification with appropriate graphical representations. The last section concludes the proposed DR detection and classification work with possible future enhancements.

\section{LITERATURE REVIEW}

This section describes the work done in the area of DR detection and classification.

Jyoti D L et al. [1] developed a method to detect DR to identify the textural changes in the retinal images using GLCM and statistical moments. A huge feature set is obtained and oversampling is performed prior to classification. Multiple classifiers like Gradient Boost, Support vector machines (SVM), AdaBoost and naïve Bayes are used to detect the presence of DR. A maximum accuracy of $88.71 \%$ and $83.34 \%$ are obtained for SVM and Random forest classifiers for the MESSIDOR database

Islam et al. [2] presented a technique for DR detection using image processing for extracting MA, blood vessels, and exudates. Student's t-test is used for feature selection. SVM is used for classification into multiple stages namely, normal, mild and moderate stages. 86.11\% accuracy percentage is reported for an online Gold standard database.

R. Venkatesan et al. [3] suggested a technique for computerized detection of retinal images for identifying MA, neovascularization as a three class problem. An enhanced color auto correlograms feature with low dimension was proposed for DR images. A multiple-instance learning framework was used to classify DR images.

Kanika et al. [4] developed a method to detect hemorrhages and vessels for the identification of DR. Analysis of density and bounding box methods are used for segmenting hemorrhages. Classification is done using random forest classifier with an overall classification accuracy of $88.75 \%$ for classifying into mild, moderate and severe cases.

Wong Li Yun et al. [5] presented a method to group the input images into multiple stages using a feed forward neural network with three layers. A total of 124 retinal images were analyzed and resulted in a sensitivity of $90 \%$.

An automated DR screening system was developed by E. Imani et al. [6] using the method of morphological component analysis. Different DR related lesions were identified by this method and the assessment outcome of the image is based on the retinal vessels. The sensitivity and specificity values recorded for MESSIDOR dataset were $92.01 \%$ and $95.45 \%$ respectively

Mane and Shreekant J [7] used an artificial neural network classifier (ANN) for DR detection. They used morphological operations with thresholding for effective segmentation of DR features. Boundaries are created around the detected areas of the retinal images for area calculation. The system achieved an accuracy of $86.36 \%$ using ANN classifier.

Identification of different stages of DR using automated techniques was proposed by Jagadish et al. [8] using random forest algorithm. This does not involve any segmentation but huge feature set was extracted and analyzed. Mazda software was used for extracting the features of the image. An accuracy of $75 \%$ was achieved using random forest classifier.

M. Gandhi and Dhanasekaran [9] used SVM classifier for the detection of DR. In this work, exudates are identified as major indicator of DR. So exudates are identified and segmented .The rigorousness of the lesions are also classified. The severity levels of DR was assessed using the support vector machine classifier 
Paing et al. [10] presented a technique for detecting lesions and classifying DR. The lesions segmented are blood vessels, MA and exudates. Various features extracted are area, count and perimeter from these lesions. All these features are fed to an ANN classifier .A total of 214 images are obtained from DIARETDB1 and other local databases.

\section{PROPOSED SYSTEM}

\section{A. Overview of the Proposed DR Detection System}

The proposed system is a multistage classification and detection of DR. The architecture of the proposed system is given in Figure 2. It comprises of image pre-processing in which the image is processed using various image processing functions to segment the MA, blood vessels, hemorrhages and exudates. This work used feature extraction step to extract a large feature set. Optimal features are selected using the attribute selected filter to select the best attributes. The selected attributes are given to a random forest classifier to grade DR into various severity levels.

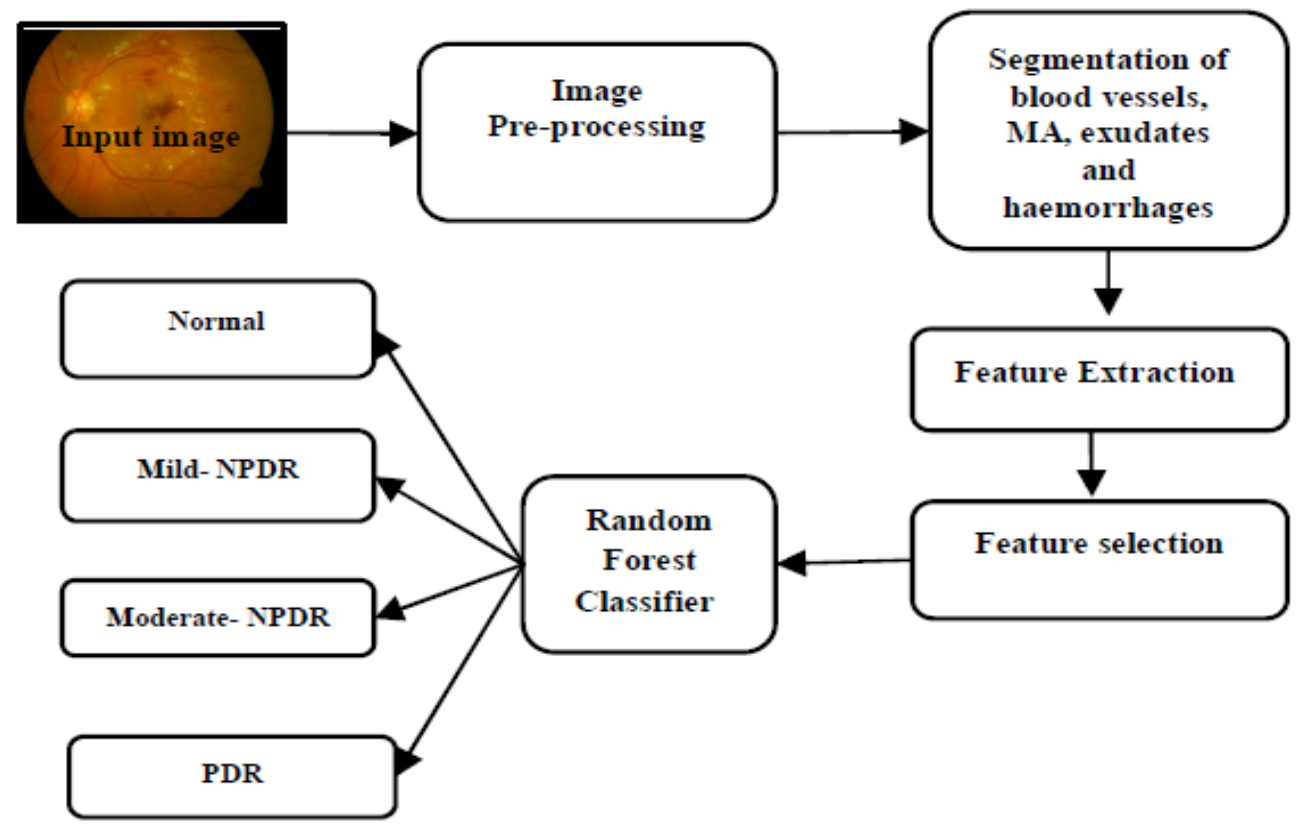

Figure 2. Architecture of the Proposed Multistage DR Detection and Classification System

\section{B. Image Pre-processing}

The purpose of image pre-processing is to make sure that the input images are suitable for extracting the retinal structures. The RGB image is converted to grey scale image. The three channels of the RGB image are extracted and analyzed. Green channel is selected as the anomalies are properly visible in this channel. This image is rescaled to $560 \mathrm{X} 720$ standard size and the aspect ratio is preserved.

The noise present in the image is separated using a median filter. The purpose of use median filter is because it restores edges yet removes noise effectively. It works by scanning through the image pixel after pixel and replaces each pixel value with the neighboring pixels value. The contrast of the image is improved using contrast limited adaptive histogram equalization (CLAHE) technique. This results in an enhanced image. This image is then subjected to segmentation.

\section{Segmentation}

The proposed work segments blood vessels, MA, exudates and hemorrhages using morphological operations and segmentation techniques.

- Segmentation of vessels: Separation of blood vessels is a major step in the detection of red lesions as the color of blood vessels, MA and hemorrhages are all red in color. The green channel image is complemented and CLAHE is performed on this image. Top hat transformation with a suitable threshold value is used with a disc shaped structuring element to segment the vessels. The resulting image comprises of blood vessels along with MA and hemorrhages and other structures. The image containing only blood vessels is obtained by using a suitable threshold value that would remove all the structures that have area less than the threshold value. 
- MA and Hemorrhage segmentation: The boundary of the fundus image is removed by generating a mask. The MA and hemorrhages are segmented by considering their circular shape and sharp texture features. Edge detection is done using the Canny edge detector and the broken edges are filled using morphological close operation using a disc shaped structuring element. This image contains vessels, MA and hemorrhages. The image containing only MA and hemorrhages is obtained my subtracting this image from the detected blood vessel image. MA and hemorrhages are further segmented based on the size using a suitable threshold value. Structures bigger than MA are classified as hemorrhages. Any fragment of blood vessel retained is removed by using a morphological open operation.

\section{Feature Extraction}

After segmenting the retinal structures, the next step is feature extraction. Various statistical attributes are obtained from the grey scale image. The textural features are obtained with the help of Gray level Cooccurrence matrix (GLCM). Texture refers to the physical structure characteristic like coarseness or smoothness. Textural features can be spectral, statistical or structural. The statistical features obtained are entropy , mean, third moment and standard deviation. They are all computed using the equations given in [12].

o Mean : The mean of an image (gray level) refers to average of all pixel values

o Standard deviation: It is the most popularly used metric which tells how much variation is found from the mean. Its low value signifies the amount of closeness of the data points to the mean value and high value indicated that the points are distributed over a large area.

o Entropy: Entropy measures the error or disorder in the data of a gray scale image. It measures the randomness of the texture in the image.

o Third moment: This feature is a measure of the skewness in the histogram of the image.

o GLCM: The second order statistical features can be extracted using the GLCM matrix. The matlab function for computing GLCM is used to compute all the features and the result is returned as a structure.

E. Feature selection using Attribute Selection Filter

The extracted features are selected using the attribute selection filter. This filter implementation allows different search and evaluation methods to be chosen. In the proposed work, Best First search method is used for selecting the attributes.

\section{F. Classification}

The selected attributes are inputted to the random forest classifier for classifying them into one of the four stages given below. The retinopathy grading of the severity levels for multistage classification of DR is presented in Table I.

Table I. Retinopathy grading rule for multistage dr classification

\begin{tabular}{|l|l|l|}
\hline Grade & Condition & Severity \\
\hline 0 & $(\mathrm{MA}=0)$ AND (Hemorrhages $=0)$ & Normal \\
\hline 1 & $(0<\mathrm{MA}<=5)$ AND (Hemorrhages $=0)$ & Mild-NPDR \\
\hline 2 & $\begin{array}{l}((5<\mathrm{MA}<15) \text { OR }(0<\text { Hemorrhages }<5)) \text { AND } \\
(\text { NeoVascularization }(\mathrm{NV})=0)\end{array}$ & Moderate -NPDR \\
\hline 3 & $(\mathrm{MA}>=15)$ OR (Hemorrhages $>=5)$ OR $(\mathrm{NV}=1)$ & PDR \\
\hline
\end{tabular}

The random forest classifier is one of the best tree based method for classification. The basic strategy is that a group of weak attributes can join together to form a strong attribute. Single decision trees often suffer from high variance problem. The steps for random forest classification is given in Table II

Table II. Algorithm for random forest classifier

Input: Feature Set obtained from Feature selection method

Output: Severity Levels normal, mild-NPDR, Moderate-NPDR and PDR

Step 1: let $T$ be the total number of trees to be built for each of the I iterations

Step 2: if the number of cases in the training set is $M$, then choose and sample $M$ cases randomly but replace with the original set. This would serve as the training set for the tree growing.

Step 3: randomly select $p$ predictors at each node. Select the best possible split. This value of $p$ is kept constant during the growing of random forest.

Step 4: do not allow pruning of the tree and grow the tree to the maximum possible extent.

Step 5: output the total prediction as the maximum vote in case of classification problem. 


\section{Results}

A. Results of Image Pre-processing and Segmentation

The steps for image pre-processing and segmentation are discussed in section III. The results of this phase are shown in Figure 3.

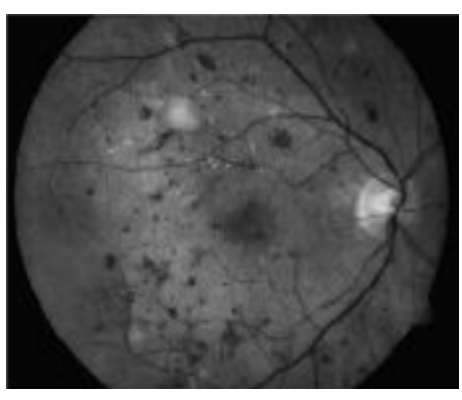

(a)

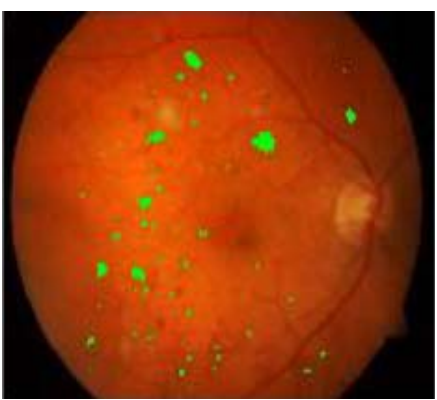

(b)

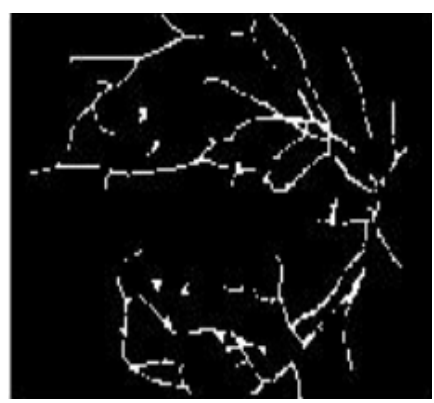

(c)

Figure 3. (a) Intensity Adjusted Image (b) Segmented Hemorrhages (c) Segmented Blood Vessels

\section{B. Performance Analysis}

Classification was done using Random forest classifier and 249 images were randomly picked from DIARETDB1 [13] and MESSIDOR [14] databases. WEKA (Waikato Environment for Knowledge Analysis) was used for classification.

Confusion Matrix: A four class classification is utilized to grade the images as normal, mild-NPDR, Moderate-NPDR and PDR. The confusion matrix is given in Table II.

Table III. Confusion matrix for random forest classifier

\begin{tabular}{|l|l|l|l|l|}
\hline CLASS & NORMAL & MILD-NPDR & MODERATE- NPDR & PDR \\
\hline NORMAL & 82 & 1 & 2 & 4 \\
\hline MILD-NPDR & 2 & 1 & 1 & 0 \\
\hline MODERATE-NPDR & 5 & 0 & 60 & 3 \\
\hline PDR & 2 & 0 & 2 & 84 \\
\hline
\end{tabular}

The average precision and recall values obtained were 0.908 and 0.912 respectively for the random forest classifier.

Accuracy: The accuracy of classification refers to the percentage of images correctly categorized as normal, mild-NPDR, Moderate-NPDR or PDR. 91.2\% accuracy was obtained by proposed method.

Receiver Operating Characteristics (ROC): The ROC graph of True Positive rate (Sensitivity) versus False Positive rate (Specificity) for the classes normal (0), mild-NPDR (1), moderate-NPDR (2) and PDR (3)are depicted in Figure 4a,b,c, and d respectively. 


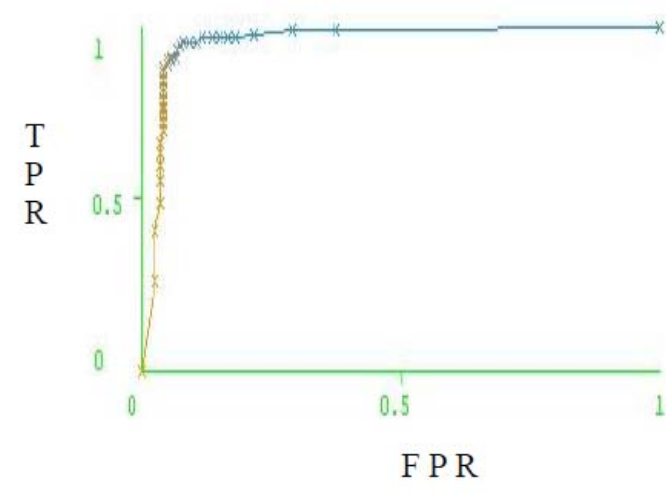

(a) ROC plot for grade 0 (normal)

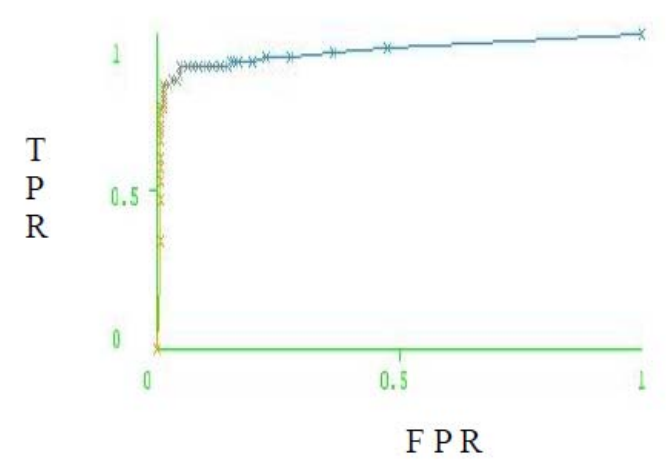

(c) ROC plot for grade 2( moderate-NPDR)

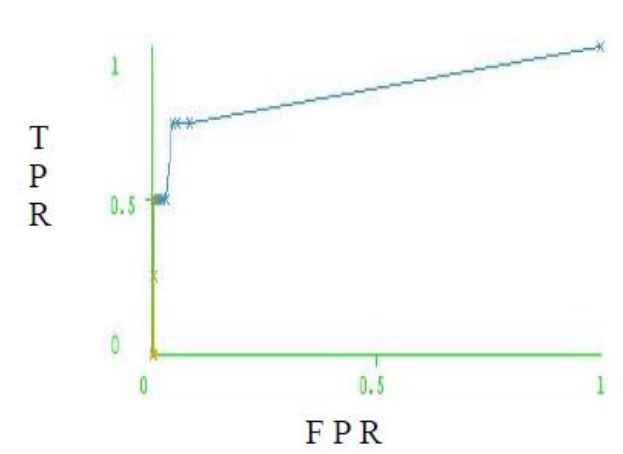

(b) ROC plot for grade 1 (mild-NPDR)

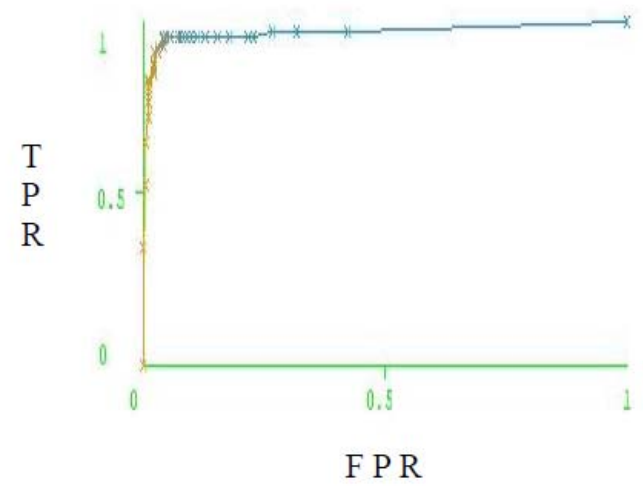

(d) ROC plot for grade 3(PDR)

Figure 4. ROC Plot for Different Severity Levels of DR for Proposed Method

The proposed method shows better results when compared to the earlier methods in terms of increased accuracy as shown in Figure 5.

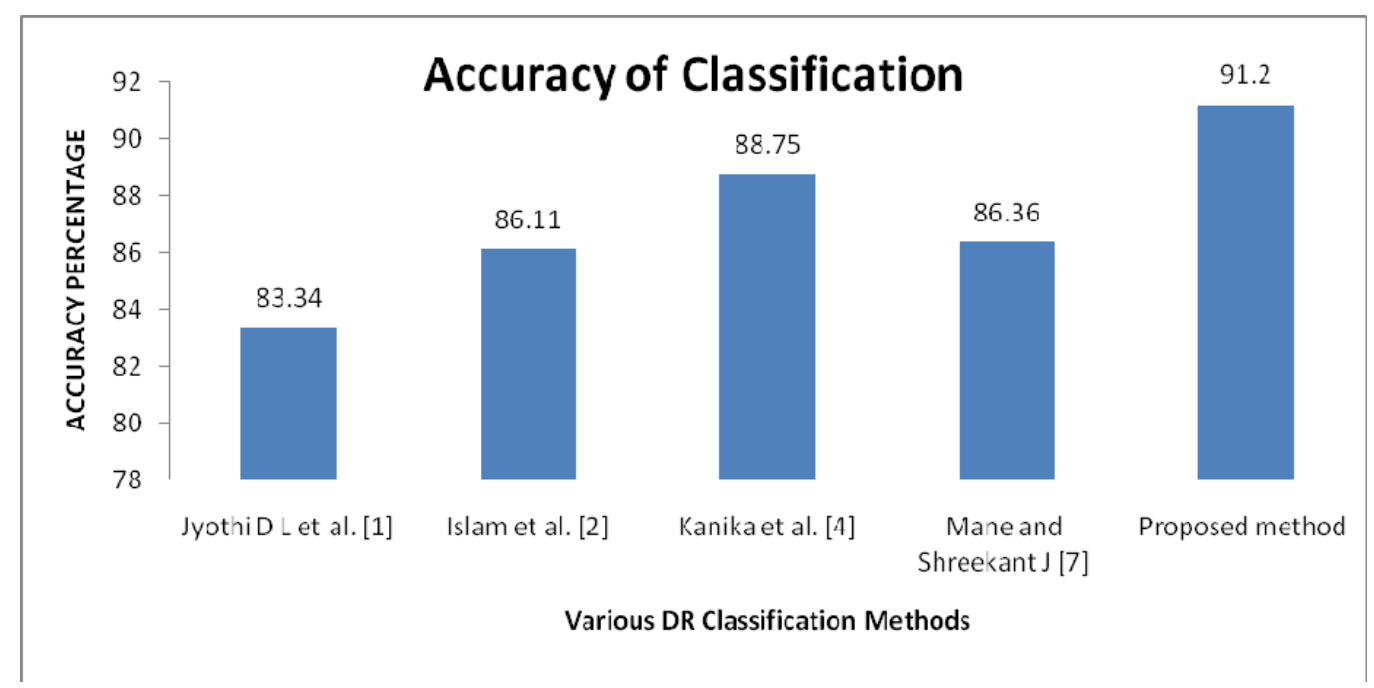

Figure 5 Performance Analysis of Various Multistage DR Detection Methods 


\section{CONCLUSION}

Diabetic retinopathy (DR) is a disorder associated with repercussion of diabetes mellitus. This would lead to blindness if not identified and treated at appropriate stages. This work proposes an automated method for multistage classification of DR using Random forest classifier. A total of 249 images are classified as normal, mild-NPDR, moderate-NPDR and PDR depending on the severity level of the disease. Input image is preprocessed and segmented using various morphological operations and thresholding techniques to segment MA, hemorrhages and blood vessels. Statistical features are extracted from these images and attribute based feature selection is used to select optimal features. These features are fed to Random forest classifier. An accuracy of $91.2 \%$ is obtained with the average precision and recall values being 0.908 and 0.912 respectively. The proposed method outweighs the earlier methods described in the literature in terms of increased accuracy. As a future enhancement, more number of images from heterogeneous databases may be considered and better image pre-processing techniques might be used to improve the accuracy.

\section{REFERENCES}

[1] J. D. Labhade, L. K. Chouthmol and S. Deshmukh, "Diabetic retinopathy detection using soft computing techniques," 2016 International Conference on Automatic Control and Dynamic Optimization Techniques (ICACDOT), Pune, 2016, pp. 175-178. doi: 10.1109/ICACDOT.2016.7877573

[2] Islam, Rafiqul \& Nur Alam, Md \& , Al-Imran-Romio \& Karim, Rezaul \& Ahmed, Khawza., “ Detection and Multi-stage Classification of Diabetic Retinopathy by Using Image Processing with SVM.”, In the Procedings of The International Conference on Biomedical System, Signals and Images ,Dec, 2012.

[3] R. Venkatesan, P. Chandakkar, B. Li and H. K. Li, "Classification of diabetic retinopathy images using multi-class multiple-instance learning based on color correlogram features," 2012 Annual International Conference of the IEEE Engineering in Medicine and Biology Society, San Diego, CA, 2012, pp. 1462-1465. doi: 10.1109/EMBC.2012.6346216

[4] K. Verma, P. Deep and A. G. Ramakrishnan, "Detection and classification of diabetic retinopathy using retinal images,"2011 Annual IEEE India Conference, Hyderabad, 2011, pp. 1-6. doi: 10.1109/INDCON.2011.6139346.

[5] Wong Li Yun, U. Rajendra Acharya, Y.V. Venkatesh, Caroline Chee, Lim Choo Min, E.Y.K. Ng, Identification of different stages of diabetic retinopathy using retinal optical images, Information Sciences, Volume 178, Issue 1,pp 106-121,ISSN 0020-0255, 2008, https://doi.org/10.1016/j.ins.2007.07.020.

[6] Elaheh Imani, Hamid-Reza Pourreza, Touka Banaee, "Fully automated diabetic retinopathy screening using morphological component analysis”, Computerized Medical Imaging and Graphics, Volume 43, Pp. 78-88, ISSN 0895-6111, 2015, https://doi.org/10.1016/j.compmedimag.2015.03.004.

[7] Mane, Shreekanth J, "Diabetic Retinopathy: Patient Identification and Measurement of the Disease using ANN", International Journal of Technical Research and Applications, e-ISSN: 2320-8663, Issue 31, pp. 278-282, 2015.

[8] Nayak J, Bhat PS, Acharya R, Lim CM, Kagathi M., "Automated identification of diabetic retinopathy stages using digital fundus images”, J Med Syst Apr;32(2):107-15, 2008, PMID: 18461814

[9] M. Gandhi and R. Dhanasekaran, "Diagnosis of diabetic retinopathy using morphological process and SVM classifier," 2013 International Conference on Communication and Signal Processing, Melmaruvathur, 2013, pp. 873-877. doi: 10.1109/iccsp.2013.6577181

[10] M. P. Paing, S. Choomchuay and M. D. Rapeeporn Yodprom, "Detection of lesions and classification of diabetic retinopathy using fundus images," 2016 9th Biomedical Engineering International Conference (BMEiCON), Laung Prabang, 2016, pp. 1-5. doi: 10.1109/BMEiCON.2016.7859642

[11] World Health Organization. Global report on diabetes. Geneva: World Health Organization; 2016

[12] D. K. Prasad, L. Vibha and K. R. Venugopal, "Early Detection of Diabetic Retinopathy from Digital Retinal Fundus Images," 2015 IEEE Recent Advances in Intelligent Computational Systems (RAICS), Trivandrum, 2015, pp. 240-245. doi: 10.1109/RAICS.2015.7488421

[13] DIARETDB1 - Standard Diabetic Retinopathy Database, "http://www2.it.lut.fi/project/imageret/diaretdb1/index.html”

[14] Decencière et al., "Feedback on a Publicly Distributed Database: The Messidor Database”, Image Analysis \& Stereology, v. 33, n. 3, p. 231-234, ISSN 1854-5165. available at: http://www.ias-iss.org/ojs/IAS/article/view/1155, 2014.

\section{AUTHORS PROFILE}

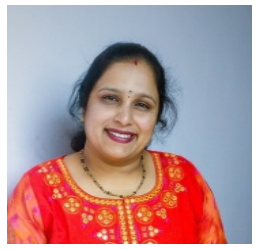

Deepthi K Prasad is currently pursuing her full time Ph.D under the guidance of Dr. Vibha L at the department of CSE, B N M Institute of Technology, Bangalore, India. She has completed her Bachelors degree and Master of Technology in Computer science and Engineering from Vishveswaraya Technological University, Belagavi, Karnataka, India. Her research interest include medical data mining, Image processing and Artificial Intelligence 

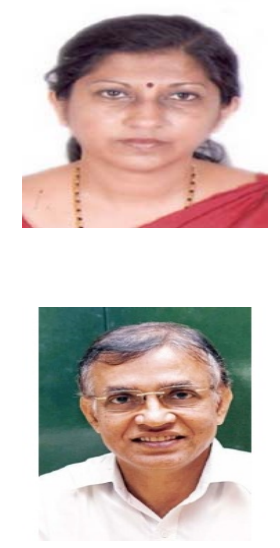

Dr. Vibha L is currently working as a Professor at the department of CSE, B N M Institute of Technology, Bangalore, India. She has completed her Bachelors degree and Master's degree from Bangalore University, Bangalore and Ph.D from Dr. MGR Educational and Research Institute, Chennai, India. Her research interest include Multimedia mining, operating system, computer architecture and digital image processing

Dr. Venugopal K R has been the Principal, University Visvesvaraya College of Engineering (UVCE). He has obtained Eleven degrees including two Ph.Ds, one in Economics from Bangalore University and another in Computer Science and Engineering from IIT Madras. He received the ME degree in Computer Science from Indian Institute of Science, Bangalore, Bachelor of Engineering in Electronics and Communications from University Visvesvaraya College of Engineering. Prof. Venugopal K R is a recipient of over Seventy Five awards, and has been conferred the prestigious IEEE Fellow Award for contributions to Computer Science and Electrical Engineering Education and ACM Distinguished Educator Award for contributions to Computer Science and Engineering Education both from USA. His research interest include Data structures, Data Mining, Computer Networks and Image processing 